\title{
DE LACROIX A CAUCHY: LA FUNDAMENTACIÓN DEL CÁLCULO INFINITESIMAL EN JOSÉ MARIANO VALLEJO (1807-1832)
}

\author{
Elena Ausejo y Francisco Javier Medrano Sánchez \\ Universidad de Zaragoza \\ ichs@unizar.es
}

Recibido: 22 de septiembre de 2014. Aceptado 10 de diciembre de 2014. Cómo citar este artículo/Citation: Ausejo, Elena y Medrano Sánchez, Francisco Javier (2015), “De Lacroix a Cauchy: La Fundamentación
del cálculo infinitesimal en José Mariano Vallejo (1807-1832)”, Asclepio, 67 (2): p113. doi: http://dx.doi.org/10.3989/asclepio.2015.31

RESUMEN: Este artículo estudia los aspectos relativos a la fundamentación del cálculo infinitesimal en la obra de José Mariano Vallejo (1779-1846), el matemático de mayor difusión en lengua española de la primera mitad del siglo XIX. Para ello, se consideran los fundamentos analíticos de su Memoria sobre la curvatura de las líneas en sus diferentes puntos, sobre el radio de curvatura y sobre las evolutas (1807) y de las dos ediciones (1813 y 1832) del volumen de su Tratado Elemental de Matemáticas dedicado al cálculo infinitesimal. El estudio muestra la dinámica evolutiva del pensamiento de Vallejo al ritmo de las referencias europeas -especialmente francesas- que encabezan el desarrollo del análisis matemático en su época y cómo conjuga la influencia sucesiva de Lagrange, Lacroix y Cauchy atendiendo a criterios de adecuación didáctica y de rigor matemático. En particular, se determinan las influencias y discrepancias en cuanto al cálculo infinitesimal entre el Tratado de Vallejo (1813) y el Traité élémentaire de calcul différentiel et de calcul intégral de Lacroix (1802), así como el alcance de la introducción de las ideas de Cauchy, expuestas por primera vez en España en la segunda edición del cálculo infinitesimal del Tratado de Vallejo (1832).

PALABRAS CLAVE: Cálculo infinitesimal; Siglo XIX; Vallejo; Lacroix; Cauchy.

\section{FROM LACROIX TO CAUCHY: THE FOUNDATIONS OF INFINITESIMAL CALCULUS IN JOSÉ MARIANO VALLEJO (1807-1832)}

ABSTRACT: This paper studies the foundations of infinitesimal calculus in the work of José Mariano Vallejo (1779-1846), the most influential mathematician in Spanish during the first half of the 19th century. For this purpose, the analytical foundations of his three main works related to infinitesimal calculus are considered: Memoria sobre la curvatura de las líneas en sus diferentes puntos, sobre el radio de curvatura y sobre las evolutas (1807), and the two editions (1813 and 1832) of the volume of his Tratado Elemental de Matemáticas devoted to calculus. The study shows the evolutionary dynamics of Vallejo's thought, following the European leading references in the development of mathematical analysis in his time, and how Vallejo combines the successive influence of Lagrange, Lacroix, and Cauchy, according to criteria of educational adequacy and mathematical rigor. In particular, the influences and discrepancies on infinitesimal calculus between Vallejo's Treatise (1813) and Lacroix' Traité élémentaire de calcul différentiel et de calcul intégral (1802) are determined, as well as the scope of the introduction of Cauchy's ideas, that were published for the first time in Spain in the second edition of the volume on infinitesimal calculus of Vallejo's Treatise (1832).

KEY WORDS: Infinitesimal Calculus; 19th Century; Vallejo; Lacroix; Cauchy.

Copyright: () 2015 CSIC. Este es un artículo de acceso abierto distribuido bajo los términos de la licencia Creative Commons Attribution-Non Commercial (by-nc) Spain 3.0. 


\section{INTRODUCCIÓN}

José Mariano Vallejo (1779-1846) es un autor de reconocida relevancia en la historia de las matemáticas en España fundamentalmente en virtud de su Tratado Elemental de Matemáticas (Vallejo, 18121817). Se trata de una obra escrita en el contexto del desarrollo de las constituciones de 28 de julio de 1799 del Real Seminario de Nobles de Madrid -institución de la que Vallejo era catedrático de Matemáticas, Ataque, Fortificación y Defensa desde $1802^{1}-$ que evolucionó al compás del equilibrio inestable que su autor sostuvo entre su actividad matemática y su compromiso político liberal ${ }^{2}$. Redactada entre 1804 y 1807, la Guerra de la Independencia retrasó el inicio de su publicación hasta 1812, lo que permitió a Vallejo actualizar sus contenidos, una práctica que se convirtió en hábito en las sucesivas ediciones del Tratado, jalonadas de notas y ampliaciones novedosas atendiendo a los desarrollos de los principales autores europeos del momento.

Aunque el análisis detallado de una obra matemática de la magnitud y modernidad de Vallejo está todavía pendiente ${ }^{3}$, sí se ha abordado el estudio del cálculo infinitesimal (Medrano, 1998), disciplina matemática que concentró los mayores esfuerzos de la comunidad matemática internacional desde su formulación en versión newtoniana o leibniciana a finales del siglo XVII hasta quedar rigurosa y sólidamente fundamentada en el primer tercio del siglo XIX sobre la base del concepto de límite de Cauchy. En este ámbito, el Tratado de Vallejo desplazó a los de su maestro Benito Bails (1731-1797), con los que se había formado en la Real Academia de Bellas Artes de San Fernando de la mano de Antonio Varas. Como quiera que los datos disponibles permiten establecer una genealogía en el proceso de consolidación del cálculo infinitesimal en España desde Jorge Juan hasta José Chaix (17651809) y Vallejo pasando por Bails y Varas, en la que cada protagonista se reconoce expresamente como discípulo de su antecesor (Ausejo y Medrano, 2015), cabe destacar que el hecho de que en sólo un par de décadas las obras de cálculo infinitesimal de Chaix (Chaix, 1801) y Vallejo superaran a las de Bails muestra el dinamismo, la permeabilidad y la capacidad de innovación adquirida por la pequeña comunidad matemática española. Que el uso del Tratado de Vallejo se prolongara hasta 1860 indica, sin perjuicio de su validez científica y didáctica, que este dinamismo no halló continuidad a lo largo del siglo XIX, lo que sitúa a Vallejo como el matemático de mayor difusión en lengua española hasta mediados del siglo XIX.
Antes de elaborar su Tratado, Vallejo ensayó sus habilidades metodológicas y didácticas con la publicación de tres obras, entre las que se encuentra la Memoria sobre la curvatura de las líneas en sus diferentes puntos, sobre el radio de curvatura y sobre las evolutas (Vallejo, 1807), de fuerte contenido científico y aspectos interesantes en cuanto al uso de elementos de cálculo diferencial, que son analizados en este artículo ${ }^{4}$.

Al cálculo infinitesimal dedicó Vallejo el cuarto volumen del Tratado Elemental de Matemáticas (Vallejo, $1812-1817$, tomo II, 2a parte). Impreso en 1813 , constituye un hito en la historia de esta disciplina en España al adoptar un enfoque de matriz euleriana, seguir a D’Alembert en la definición del cálculo mediante el concepto de límite y reducir el cálculo infinitesimal a álgebra al estilo de Lagrange (Medrano, 1998, pp. 958-961). Este artículo profundiza en el estudio de esta obra analizando cómo el cálculo infinitesimal de Vallejo introduce a Sylvestre-François Lacroix (17651843) en España ${ }^{5}$, siguiendo - aunque con discrepancias importantes - su Traité élémentaire de calcul différentiel et de calcul intégral (Lacroix, 1802; 1806), probablemente el libro de texto de cálculo infinitesimal mas difundido y traducido del siglo XIX.

La segunda edición del cuarto volumen del Tratado Elemental de Matemáticas (Vallejo, 1832) -corregido y aumentado, pasando de 252 a 503 páginas - fue resultado directo de la intensa actividad formativa de Vallejo durante su exilio parisino (1825-1829), donde tuvo la oportunidad de actualizar sus conocimientos matemáticos asistiendo a los cursos de Lacroix, Laplace, Cauchy y Gay-Lussac - con quienes pudo establecer relación personal- (Hernanz y Medrano, 1990, pp. 435-437). En este artículo se analiza la incorporación al Tratado de los nuevos conocimientos adquiridos en el exilio en el terreno del cálculo infinitesimal, especialmente la influencia de las ideas de Cauchy, que fueron expuestas por primera vez en España ${ }^{6}$.

\section{MEMORIA SOBRE LA CURVATURA DE LAS LÍNEAS EN SUS DIFERENTES PUNTOS, SOBRE EL RADIO DE CURVATURA Y SOBRE LAS EVOLUTAS (1807)}

En esta Memoria Vallejo se plantea el estudio de evolutas y radios osculadores al objeto de preparar el Tratado que le había sido encargado por el director del Real Seminario de Nobles, una obra de amplia envergadura cuya mayor dificultad consistía, en opinión Vallejo, en el hecho de que los principios de las matemáticas aún no se hallaban en estado de «poderse exponer completamente» en una obra elemental (Va- 
Ilejo, 1807, p. 4). Ciertamente, en un periodo de ampliación del campo numérico, de discusión en torno a los fundamentos del cálculo infinitesimal y de desarrollo de su interacción con el álgebra y la geometría, que discurría paralelamente al proceso de establecimiento del alcance social y los contenidos doctrinales de la enseñanza primaria, secundaria y superior, no había referentes sólidos y seguros ${ }^{7}$ sobre los que Vallejo pudiera adecuar la amplitud y la complejidad de los contenidos matemáticos a sus estudiantes: «jóvenes de trece á catorce años, que es la edad media á que principian á estudiar estas ciencias los caballeros Seminaristas» (Vallejo, 1807, p. 5).

Los primeros frutos del trabajo de Vallejo fueron las Adiciones a la Geometría de D. Benito Bails (1806) y la Aritmética para niños escrita para uso de las escuelas del Reyno (1806), obras de preparación del Tratado que estaba desarrollando (Vallejo, 1807, pp. 6-7). En este proceso observó que el tema de evolutas y radios osculadores estaba «bastante inconexo» (Vallejo, 1807, p. 7), lo que le llevó a prepararlo y desarrollarlo de manera completa y «siendo esto ya mas de lo que debía contenerse en una obra elemental» (Vallejo, 1807, p. 8), decidió desgajarlo del Tratado y publicarlo en forma de Memoria.

Tras un amplio desarrollo histórico del problema, que abarca todo el abanico temporal desde Huygens hasta culminar con Euler, Monge, Lagrange Laplace, Chaix y Lacroix (Garma, 1994, pp. 230-231), Vallejo pasa a trazar el plan de su obra, en la que destacan algunos puntos interesantes sobre el uso de elementos de cálculo diferencial.

Comienza su exposición definiendo curvatura de una línea curva para, una vez establecida la definición que le parece más exacta y fácil de manejar, pasar a encontrar la fórmula matemática para calcular su valor. Considera "la línea como originada del movimiento de un punto, la superficie del movimiento de una línea y el volumen, ó el cuerpo geométrico del movimiento de una superficie» (Vallejo, 1807, p. 22). Estas concepciones presentan un espíritu newtoniano en el manejo de curvas en cuyo planteamiento aparecen componentes mecánicas que posteriormente rechazará en su Tratado, al criticar el planteamiento de Newton por hacerlo "valiéndose de las ideas de movimiento que son extrañas en la análisis (sic)» (Vallejo, 1812-1817, tomo II, 2a parte, p. 110). Su uso puede entenderse como lícito en una obra que pretende resolver el problema de las evolutas usando cualquier argumentación válida y no restringida a procedimientos analíticos -en cualquier sentido de la palabra-, dado que en este momento histórico todos los autores tienen una idea dinámica de una curva, en la que algo se mueve, en relación al tiempo o a una sucesión de valores de la variable. Esta concepción no sólo contamina la idea de curva sino también la de función, con expresiones sencillas pero equívocas, como «la variable recorre una sucesión de valores».

Más adelante definirá polígono como la línea originada por un punto que cada cierto tiempo varía de dirección y, como le interesan las líneas curvas, afirmará: «si pasamos ahora á los límites, esto es, si consideramos que el punto A varía á cada instante de dirección, entonces en vez de trazar un polígono $A B C D E$, trazará una curva AFH» (Vallejo, 1807, p. 24). Subyace aquí la idea de que una curva es en realidad un polígono de infinitos lados infinitesimales, idea entonces debatida sobre la que no existía consenso ${ }^{8}$.

Seguidamente, para hallar la fórmula de la curvatura de un arco calculada en un punto de la curva, hace un estudio local de la curva y trabaja con consideraciones infinitesimales. Hasta este momento ha usado el concepto de curva como la trayectoria de un punto en movimiento, pero ahora cambia radicalmente su planteamiento e introduce todo el aparato analítico necesario con sus correspondientes presupuestos teóricos: las curvas son tratadas como funciones $z=f, x ; u=f$, $t$ (Vallejo, 1807, pp. 33, 44, $89)^{9}$, lo que permite usar las herramientas del análisis - coeficiente diferencial, teorema de Taylor, etc. -

Como quiera que en toda esta parte Vallejo utiliza como referencia las Instituciones de Chaix (1801) que cita varias veces-, sus exposiciones están basadas en derivadas (coeficientes diferenciales), no en diferenciales ${ }^{10}$. Así, basará el estudio de los contactos entre dos curvas en sus respectivos desarrollos de Taylor, aun siendo consciente de que existen ocasiones en los que este desarrollo no sirve: "la análisis (sic) manifiesta que cuando algún valor de $x$ hace infinitos los coeficientes diferenciales $d z / d x, d^{2} z / d x^{2}, \& c$. el desarrollo de $z^{\prime}=f(x+k)$ contiene otras potencias que $k, k^{2}, \& c$. y en este caso no tiene lugar el método que acabamos de explicar» (Vallejo, 1807, p. 41).

A diferencia de otros autores, no considera este caso como una deficiencia de la fórmula de Taylor, simplemente admite que no posee la generalidad que parecen defender otros autores. Esta posición la anticipa ya en el prólogo, cuando afirma que hay valores en los que usa otro método «no teniendo lugar el teorema de Taylor» (Vallejo, 1807, p. 18). Mantendrá también esta posición en su Tratado, con lo que 
parece plantear una búsqueda de restricciones a las fórmulas o teoremas hasta entonces considerados válidos sin ninguna limitación. Con ello, Vallejo se encuadra ya en la tendencia al rigor que caracteriza al siglo XIX, cuyo máximo exponente en el primer tercio del siglo será Cauchy - por ejemplo con sus planteamientos sobre el uso de las series no convergentes. Para el caso en que no se pueda aplicar el teorema de Taylor, afirma, siguiendo a Euler, que una función en ese punto siempre se podrá escribir de la forma $f(x+k)=f(x)+A k^{\lambda}+B k^{\lambda+\mu}+C k^{\lambda+\mu+v} \operatorname{con} \lambda, \mu, v>0$.

Es destacable que Vallejo maneje coeficientes diferenciales de forma generalizada y sólo utilice diferenciales en algunos pasos concretos, por ejemplo, cuando dada la ecuación de una circunferencia $(\alpha-t)^{2}+(\zeta+u)^{2}=\gamma^{2}$ halla el valor de $d u / d t$ diferenciando la expresión anterior, con lo que obtiene $-2(\alpha-t) d t+2(\zeta+u) d u=0$ y finalmente $d u / d t=(\alpha-t) /(\zeta+u)$ (Vallejo, 1807, p. 47). No obstante, pese a que la utilización de las diferenciales estaba muy arraigada en los usos de los matemáticos del momento, los presupuestos geométricos relacionados con la diferencial están totalmente ausentes de esta Memoria. Vallejo maneja funciones en los cálculos analíticos, incluso cuando está trabajando con conceptos geométricos como curva, curvatura o círculo osculador, donde la diferencial podría haber sido usada con el cómodo concepto de elemento infinitamente pequeño de arco. Esto se manifiesta claramente, por ejemplo cuando, tras hallar la expresión del radio $\gamma$ y centro $(\alpha, \beta)$ del círculo osculador, asegura: "los valores $\alpha, \beta, \gamma$ que hemos obtenido están expresados por coeficientes diferenciales que son funciones de las coordenadas de la curva» (Vallejo, 1807, p. 54).

En esta frase expresa claramente su idea, acorde con Lagrange, de que los coeficientes diferenciales ${ }^{11}$ son funciones, lo que encuadra su posición en la corriente de algebrización del análisis, donde la función es el centro del estudio. El cálculo diferencial trata sobre funciones y no sobre conceptos geométricos como curvas o términos ambiguos como cantidades.

No obstante, la Memoria muestra también las lagunas matemáticas de Vallejo que, en un ejercicio de juvenil osadía intelectual, se atrevió a proponer para la indeterminación $0^{\circ}$ una demostración del resultado $0^{0}=1$ (Garma, 1994, p. 234) -aplicando reiteradamente la hoy conocida como regla de L'Hôpitalque constituye todo un ejemplo de las dificultades planteadas y de la laxitud metodológica en la praxis matemática del momento: mezcla casos generales y particulares, usa elementos relacionados con límites de manera soterrada y hace prevalecer la forma de las expresiones y fórmulas sobre sus propias limitaciones (Vallejo, 1807, pp. 74n y ss.). Vallejo debió advertir su error más pronto que tarde, porque omitió la cuestión en la primera edición de su Tratado y la trató adecuadamente en la segunda (Vallejo, 1832, pp. 323-325) ${ }^{12}$.

\section{LA INFLUENCIA DE LACROIX EN EL CÁLCULO INFI- NITESIMAL DE VALLEJO (1813)}

Con anterioridad a la publicación del cálculo infinitesimal de Vallejo (Vallejo, 1812-1817, tomo II, 2a parte), Lacroix había publicado dos obras sustancialmente distintas sobre cálculo infinitesimal, en primer lugar un enciclopédico Traité du Calcul différentiel et du Calcul intégral en tres volúmenes (Lacroix, 17971800 ) y posteriormente una exitosa versión abreviada del mismo que, pese a estar diseñada para los primeros cursos de enseñanza superior (Caramalho Domingues, 2008, pp. 283-285), añadía al título del primer tratado el adjetivo «elemental» (Lacroix, 1802) ${ }^{13}$. La diferencia entre ambas obras no es sólo de extensión, sino también de fundamentación: mientras en el Tratado elemental hace uso de los límites, en el Tratado mayor utiliza la teoría de Lagrange - aun reconociendo la validez de la teoría de los límites ${ }^{14}$.

Pues, bien, por lo que respecta a las fuentes del cálculo de Vallejo, puede constatarse que la principal es el Tratado elemental de Lacroix, del que toma literalmente todos los ejemplos que desarrolla en la parte dedicada al cálculo diferencial e incluso aquéllos que propone al lector, sin ni siquiera cambiar las letras con las que denomina a las constantes o el orden de los ejemplos (Vallejo, 1812-1817, tomo II, 2a parte, pp. 120-123; Lacroix 1806, pp. 17ss.; Lacroix, 1810, pp. 152ss.). Si bien estos ejemplos pueden encontrarse en ambos tratados de Lacroix, el hecho de que Vallejo se decante por los límites sí apunta claramente al Tratado elemental de Lacroix como principal fuente de Vallejo.

Las razones de Lacroix para inclinarse por el método de los límites para introducir la derivada -el coeficiente diferencial- en su Tratado elemental fueron esencialmente didácticas, concretamente de brevedad expositiva. Por el contrario, en su Tratado mayor se había alineado con Lagrange en el sentido de considerar los coeficientes diferenciales como el coeficiente del incremento en el desarrollo de una función en serie de potencias, una posición que Lagrange defendía para evitar el uso de límites y otros conceptos poco claros desde su punto de vista. 
En el caso de Vallejo las razones pudieron ser más complejas. Vallejo pertenecía a una escuela que -desde Bails (Ausejo y Medrano, 2015) hasta Chaix (Medrano, 1998, pp. 956-958) pasando por Ciscar (Ausejo y Medrano, 2012) - había ido encauzándose paulatinamente por la senda de los límites frente a las diferenciales, que Vallejo trataba de evitar pese a que Lacroix las utilizaba profusamente. Vallejo era langrangiano en cuanto que practicaba la reducción del cálculo infinitesimal al álgebra y creía -erróneamente, como Lagrange- en la existencia del desarrollo en serie de potencias de cualquier función, pero se resistía a adoptar la exposición de su Théorie de fonctions analytiques en toda su generalidad en virtud de algunos inconvenientes prácticos que nunca terminó de precisar (Vallejo, 1812-1817, tomo II, 2a parte, p. 111). En este contexto, la solución más natural pudo ser adoptar como guía el Tratado elemental de Lacroix, un texto moderno cuya fundamentación compartía y que, además, había sido expresamente concebido para el propósito docente que Vallejo tenía encomendado.

La cuestión de las diferenciales, a las que Lacroix da en su Tratado elemental carta de naturaleza fundamental en su desarrollo - calculando de forma directa las diferenciales de las funciones y equiparando la diferencial con el incremento-, hace que en el cálculo infinitesimal de Vallejo se combinen partes tomadas -traducidas - literalmente de Lacroix con otras en las que se aleja de éste. Así, en la parte dedicada a las diferenciales de orden superior, tras introducir la cuestión con un párrafo traducido de Lacroix, adopta en el desarrollo la notación lagrangiana de los apostrofes: $f, f^{\prime}, f$ ", ..., que Lacroix rechaza explícitamente (Lacroix, 1806, p. 524n) ${ }^{15}$.

La asunción de la notación y de sus funciones primeras implica también que se refuerzan las ideas de que los coeficientes diferenciales de orden superior $f^{\prime}, f^{\prime \prime}$, ... son funciones definidas de manera unívoca por la función primitiva $f$ y de que el coeficiente diferencial no es un cociente de nada. Las diferenciales no existen y son, de hecho, cero. No usa la expresión función derivada, pero habla de que la función $A$, que representa el primer coeficiente diferencial, «se deriva por una ley constante de $f .(x)$ » (Vallejo, 1812-1817, tomo II, 2a parte, p. 124). Y después vuelve a la vía expuesta por Euler y seguida por Lacroix, de manejar los coeficientes diferenciales, como $d z / d x=A$. Aplica estas relaciones para desarrollar en serie una función que ejemplifica en el caso del binomio de Newton, $(x+k)^{\mathrm{n}}$, que obtiene calculando las derivadas sucesi- vas y haciendo $x=0-\sin$ mencionar el caso del exponente irracional ${ }^{16}$ - y continúa con la demostración del teorema de Taylor siguiendo el Tratado elemental de Lacroix casi al pie de la letra (Vallejo, 1812-1817, tomo II, 2a parte, p. 128). Pero seguidamente copia una digresión de Lacroix sobre la forma en que queda la diferencia de una función a la que se le da un incremento aunque, mientras Lacroix trabaja utilizando diferenciales $(d x)$, Vallejo usa $\Delta z=z^{\prime}-z$ y $\Delta x$. De este modo deja el resultado en función de los coeficientes diferenciales, como indicando nuevamente su rechazo al manejo de diferenciales. La idea de manejar sólo coeficientes diferenciales, es decir, derivadas frente a diferenciales, se repite con insistencia a lo largo de la obra de Vallejo, que recoge así la influencia de Lagrange al seguir claramente la vía establecida por Chaix (Medrano, 1998, pp. 957-958).

Otro tanto ocurre en la deducción de la fórmula de la diferencial de la función exponencial $z=a^{x}$, donde Vallejo sigue esencialmente los pasos de Lacroix (1806, pp. 26-27) aunque recurriendo al incremento $\Delta x$ de la variable $x$ para evitar en todo momento el manejo de las diferenciales, que sólo aparecen en el resultado final: $d a^{x}=k a^{x} d x$ (Vallejo, 1812-1817, tomo II, 2a parte, p. 136).

Es destacable la coherencia que Vallejo mantiene en este aspecto, que abarca toda su obra: su definición de la diferencial es posterior a la del coeficiente diferencial (derivada) ${ }^{17}$-el único concepto que considera riguroso- y nunca parte de las diferenciales en una demostración; las operaciones se realizan con el coeficiente diferencial (derivada) y sólo una vez deducida una propiedad para los coeficientes diferenciales se traducen a diferenciales. La diferencia, aunque sutil, refleja un posicionamiento distinto frente a los fundamentos del cálculo y muestra la presencia de la idea de derivada, ya apuntada por L'Huilier, como elemento fundamental, frente al de diferencial - que aparece en muchas obras del momento. La cuestión no afecta a los resultados: prácticamente todos los autores, salvo Lagrange, admiten la diferencial como herramienta útil, pero muchos de ellos se sitúan claramente en la corriente que pretende erradicar la diferencial de la base del cálculo, entre ellos Chaix y Vallejo.

Por otra parte, cabe advertir que la estructura del tratado de Vallejo difiere de Lacroix. Vallejo lo dispone en la forma planteada por Euler, en el sentido de que las diferencias finitas preceden al estudio del cálculo diferencial -aunque las usa poco en el desarrollo posterior-, mientras que Lacroix las considera en un apéndice final, indicando que es una forma de 
presentación menos simple para las mismas ideas de fondo (Lacroix, 1806, p. 523n.). También se observa en Vallejo un apartado sobre la Teoría de los límites que preludia el rumbo que pronto va a tomar la fundamentación del cálculo infinitesimal.

A modo de resumen se puede concluir diciendo que:

- El desarrollo de Vallejo es similar al de Chaix, adoptando sus presupuestos más importantes, con el concepto de límite como elemento fundamental.

- La fuente fundamental es el Tratado elemental de Lacroix, del que toma, en ocasiones de manera literal, buena parte de su desarrollo.

- A pesar de tener como referente la obra de Lacroix, existen discrepancias importantes, sobre todo en el manejo de las diferenciales, que Vallejo evita mientras que las utiliza de manera abundante.

- También discrepa de Lacroix en el esquema general, en el que las diferencias finitas preceden al cálculo diferencial, lo que indica cierta influencia de las posiciones eulerianas.

- Hace uso de la notación y alguna demostración de Lagrange, aunque no asume su desarrollo expositivo.

\section{LA INFLUENCIA DE CAUCHY EN LA SEGUNDA EDI- CIÓN DEL CÁLCULO INFINITESIMAL DE VALLEJO (1832)}

La segunda edición del cálculo infinitesimal de Vallejo (Vallejo, 1832) no varió la estructura de la primera (Vallejo, 1812-1817, tomo II, 2a parte), pero duplicó el número de páginas. Las 250 páginas de aumento se concentraron en las partes dedicadas al método de los límites (27 pp.), cálculo diferencial (140 pp.) -especialmente aplicaciones a la determinación de máximos y mínimos (80 pp.) y teoría de líneas curvas (26 pp.) - y cálculo integral (52 pp.) -especialmente integración de funciones racionales (34 pp.).

Junto a adiciones puntuales, como la definición de la diferenciación como una operación - no explicitada en la primera edición - o la digresión sobre la exactitud de la extensión de la fórmula de derivación de $x^{n}$ a exponentes no racionales ${ }^{18}$, se incorporaba una modificación de gran calado en la presentación del cálculo diferencial: en la primera edición, aunque basada en la teoría de los límites, se necesitaba conocer el desarrollo en serie de potencias como paso previo (Medrano, 1998, pp. 959-960), mientras que en la se- gunda edición el cálculo diferencial permite calcular las derivadas sin necesidad de conocer previamente el desarrollo en serie, que se obtiene también mediante el cálculo diferencial:

[El Cálculo] tiene procedimientos directos y sencillos, para, dada una función, encontrar desde luego el límite de la relación del incremento de la función con el de la variable, sin necesidad de hallar anticipadamente ni el incremento de la función, ni la relación de este incremento con el de la variable; y lo que todavía es mas portentoso, es que el mismo Cálculo diferencial suministra medios mas expeditos que los explicados hasta aquí, para hallar la diferencia ó incremento de la función, su relación con la diferencia ó incremento de la variable, y desenvolver en series las funciones independientemente de la teoría de las series (Vallejo, 1832, p. 165) ${ }^{19}$

Este es el mensaje planteado por Cauchy ${ }^{20}$, el autor que aparece con más fuerza en esta segunda edición del cálculo infinitesimal del Tratado, como cabía esperar tras la tercera edición del segundo volumen del Tratado Elemental de Matemáticas de Vallejo, publicada en 1825, en el que aparecía la llamada al rigor frente a las razones sacadas de "la generalidad del Álgebra» hecha por Cauchy en su Curso de Análisis (Cauchy, 1821):

Mr. A.L. Cauchy, á quien tengo el honor de conocer personalmente, y con quien he tenido la satisfacción de conferenciar en París sobre varios puntos de las Matemáticas coincide tanto con mis ideas, que no puedo menos de poner aquí algunas de sus aserciones, aunque precisamente no sean todas ellas relativas á la Geometría (Vallejo, 1825, p. VI).

A mayor abundamiento, Vallejo tomaba de Cauchy la frase «los principios del cálculo diferencial y sus más importantes aplicaciones, se pueden exponer muy fácilmente sin la intervención de las series» ${ }^{21}$ para asegurar que:

como yo explico todo el cálculo diferencial sin fundar sus principios en el desarrollo de las series, resulta que me he anticipado mucho en este asunto; y si se tiene en consideración qué Mr. Cauchy supone ya conocida la fórmula del binomio de Newton, y que yo explico los principios de dicho cálculo, tanto en este tratado, como en mi compendio de Matemáticas, sin suponer demostrada la expresada fórmula, no se extrañará el que yo haya asegurado haber llevado algo mas adelante las ideas de Mr. Cauchy (Vallejo, 1825, p. VII). 
A partir de esta fecha Vallejo hizo sucesivas referencias a un total de cinco obras distintas de Cauchy, lo que parece indicar que estaba al tanto de sus publicaciones y que consideraba su obra digna de estudio. Como se verá en adelante, ello no significa que siempre asumiera sus propuestas, pero sí que supo reconocer cuál era el camino más fructífero.

\subsection{Adiciones en las que se cita a Cauchy}

Cauchy es el autor más citado en la segunda edición corregida y aumentada del volumen del Tratado Elemental de Matemáticas de Vallejo dedicado al cálculo infinitesimal, con 15 citas en diferentes temas ${ }^{22}$. A bastante distancia le sigue - con 9 citas - Lacroix $^{23}$, Poisson ${ }^{24}-7$ citas - y Euler ${ }^{25}$ y Lagrange ${ }^{26}$ - con 6 citas cada uno de ellos.

Ya al principio del volumen Vallejo advierte sobre las limitaciones impuestas por Cauchy para el manejo de las series, recomendando su obra para ampliar el estudio de este tema:

algunas veces se ha querido dar á entender que una serie, aunque divergente, podía ser empleada en cálculos analíticos, en lugar de la función de que provenía; y como los resultados que se obtienen por el intermedio de las series divergentes son siempre inciertos y el mayor número de veces inexactos, al menos en todas aquellas propiedades, que dependen de la sumación de sus términos, varios geómetras de mérito, entre los cuales ocupan el primer lugar Mr. Poisson, y Mr. Cauchy, se han esforzado para desvanecer este error; y aun se ha creído necesario restringir mas la idea de convergencia, para que una función se pueda sustituir por su desarrollo; y así, recomendamos sobre este particular la lectura del capítulo 60 del curso de análisis de Mr. Cauchy, donde establece varios teoremas para conocer la convergencia ó divergencia de las series (Vallejo, 1832, 38n).

La cita concreta del texto de Cauchy indica un conocimiento bastante preciso de su obra y no deja lugar a dudas sobre su lectura y asimilación. De nuevo es citado Cauchy como texto de consulta para ampliar todo lo relativo a las series imaginarias, convergentes y divergentes (Vallejo, 1832, 81) ${ }^{27}$.

El conocimiento de la obra de Cauchy por parte de Vallejo es además extenso: las citas aparecen en diferentes momentos a lo largo de todo el volumen y las referencias atañen a cinco obras. La mayor parte proceden del Curso de Análisis (Cauchy, 1821) - con o sin referencia explícita a la obra-, pero también aparece una referencia a las Leçons sur le Calcul différentiel a propósito de la denominación del teorema de MacLaurin, que se atribuyó a este autor en la novena lección de la obra de «Mr. Cauchy impresa en este mismo año de 1829» (Vallejo, 1832, p. 195) (Cauchy, 18821974, 2a ser., tomo IV, p. 364). El hecho de que se cite esta obra, entonces recién publicada, no sólo indica que Vallejo estaba muy bien informado de los trabajos de Cauchy, sino que sirve para acotar la fecha de esta segunda edición del cálculo infinitesimal, que habría sido elaborada, al menos parcialmente, en dicho año.

Otra cita especialmente interesante es la que se refiere a la teoría del cálculo de residuos (Vallejo, 1832, p. 338), que Cauchy desarrolló entre los años 1826 y $1830^{28}$ en una serie de trabajos publicados en sus Exercices de Mathématiques ${ }^{29}$. Vallejo menciona esta teoría al estudiar las formas indeterminadas $0^{\circ}$, $\infty^{0}, \ldots$, donde se hace alusión a la descomposición de una fracción racional en fracciones simples. El hecho de que la referencia se encuentre al margen de toda relación con la integración de funciones complejas, circunscrita a una única aplicación de la teoría del cálculo de residuos - la descomposición de una fracción algebraica en fracciones simples- muestra que Vallejo sigue la obra de Cauchy con el suficiente detalle como para referirse a una aplicación específica de la teoría de los residuos.

Vallejo cita también a Cauchy cuando refiere el contraejemplo dado por el matemático francés para demostrar que no siempre es posible intercambiar el orden de integración de las integrales dobles: dedica la mayor parte del $\$ 667$ a exponer casos en los que la permutabilidad es permitida $-\mathrm{y}$ algún otro en el que no-, expone las limitaciones impuestas por Cauchy (Vallejo, 1832, p. 471) y estudia el ejemplo expuesto en su Mémoire sur les intégrales définies, leída en la Academia de Ciencias de París en 1814 y publicada en 1827, en el primer tomo de las Mémoires de l'Académie royale des sciences de l'Institut de France (Cauchy, 1882-1974, 1a ser., tomo I, pp. 320-506, ejemplo pp. 388ss.).

Por último, Vallejo ofrece la definición de integral singular dada por Cauchy en la lección 25 de su Résumé des leçons données à l'École Polytechnique sur le calcul infinitésimal (Cauchy, 1823, p. 97) (Vallejo, 1832, p. 474).

\subsection{El concepto de continuidad}

El edificio matemático levantado por Cauchy está cimentado en dos conceptos clave que le servirán para romper con toda una tradición algebraica representa- 
da en su vertiente más radical por Lagrange y su intento de reducir el análisis al álgebra a través del desarrollo de Taylor de las funciones. Cauchy pulveriza estas ilusiones dando estatus separado al análisis mediante los conceptos de continuidad y convergencia, al demostrar que estos conceptos no pueden ser tratados por procedimientos únicamente algebraicos.

La importancia del concepto de continuidad en Cauchy se constata en la cantidad de veces que utiliza la condición de continuidad en sus obras. No es un concepto más de su trabajo, sino el elemento aglutinador de su teoría (Cauchy, 1994, p. 47). Por tanto, no cabe sino abordar inexcusablemente si Vallejo comprendió el cambio que estaba planteando el autor francés o continuó manejando la idea intuitiva de continuidad entonces tan extendida, que presuponía un comportamiento "bueno" de las funciones (continuidad, derivabilidad, ...) en virtud de una cierta «ley de continuidad».

Desde luego conocía las definiciones de Cauchy, que aparecen copiadas del Curso de Análisis (Cauchy, 1821) - sin cita expresa y con alguna matización interesante - en la parte dedicada al cálculo de las diferencias de funciones. Así, cuando define el concepto de función continua, que toma de Cauchy, asegura:

cuando en una función $f(x)$, que tiene una valor único y finito para todos los valores de $x$ comprendidos entre dos límites dados, la diferencia $f(x+k)-f(x)$ es siempre, entre éstos límites, una cantidad muy pequeña, se dice que $f(x)$ es función continua de la variable $x$ entre los límites ${ }^{30}$ de que se trata (Vallejo, 1832 , p. 154$)^{31}$.

Cabe observar que no hace mención alguna del papel que representa $k$, de manera que su definición carece de sentido al omitir la relación entre los incrementos de la variable y los de la función. No ocurre lo mismo en Cauchy, cuya definición es clara y menciona dos veces la idea de que un incremento pequeño de la variable produce un incremento pequeño de la función (Cauchy, 1821, pp. 34-35). Puede deberse a una errata o a un error de redacción, ya que poco más adelante, al estudiar la continuidad de la función $f(x)=1 /(5-x)$ entre los límites 0 y 4, Vallejo toma el ejemplo de $x=2$ y $k=0,1$ y, tras obtener $f(x+k)-f(x)=1 / 87$, afirma: «y mientras más pequeño supongamos el valor de $k$, menor será el de $f(x+k)-f(x) »$ (Vallejo, 1832, p. 154), idea correcta que es la expresada por Cauchy en su definición.

Después de dar esta primera definición de Cauchy traduce dos párrafos en los que introduce el con- cepto de función continua en un entorno ${ }^{32}$ de un valor y el de función discontinua o que tiene solución de continuidad. El concepto de función discontinua ya lo ha usado Vallejo con anterioridad al trabajar con los valores aislados de las funciones. Según él, las funciones en las que hay valores reales separados de valores imaginarios se llaman funciones discontinuas y "hay solución de continuidad ${ }^{33}$, en las funciones, siempre que, para valores sucesivos de la variable, resulten interrupciones de valores reales, y de valores imaginarios en los de la función» (Vallejo, 1832, p. 4).

Seguidamente explica el caso en que para algún valor de la variable la función se hace infinita, en el que también afirma que hay "solución de continuidad».

Este estudio de los puntos aislados y de valores infinitos le lleva a asegurar cuando estudia la continuidad que estos son los dos únicos casos posibles de discontinuidad (Vallejo, 1832, p. 155).

Aquí termina la parte dedicada a la continuidad tomada de la obra de Cauchy. No hace, sin embargo, ningún estudio completo de las funciones elementales como Cauchy, que da una relación de qué funciones son continuas y entre qué límites. Así, detecta la importancia del concepto de continuidad al añadirlo en la segunda edición, pero sin repercusiones importantes en el desarrollo posterior de la obra: el papel de la continuidad como concepto por el que pasan los resultados más importantes del análisis de Cauchy está ausente de la obra de Vallejo.

Esta ausencia no desmerece la capacidad de VaIlejo para vislumbrar los conceptos esenciales que a la postre abrirían nuevas vías de trabajo altamente fructíferas si se considera debidamente contextualizada en los tiempos de edición y en las características del Tratado de Vallejo, que es elemental en el título pero de nivel medio en sus contenidos - con algunos temas que resultan tremendamente difíciles para los no iniciados-; a esta obra incorpora Vallejo en su segunda edición conceptos y resultados obtenidos en la década inmediatamente anterior sin alterar consecuentemente la estructura del volumen, básicamente porque el encaje docente de los nuevos fundamentos del análisis no podía hacerse sin un proceso previo de asimilación. De hecho, Vallejo fue el único autor hispano de la primera mitad del siglo XIX que incluyó estas novedades, que tampoco fueron consideradas por autores franceses de amplia difusión docente, como Lacroix, Boucharlat o Francoeur. 


\section{CONCLUSIONES: LACROIX Y CAUCHY EN EL CÁLCULO INFINITESIMAL DE VALLEJO, ENTRE LA DIDÁCTICA Y EL RIGOR MATEMÁTICO}

La figura de Cauchy no fue excesivamente bien vista por sus coetáneos: su carácter difícil y sus ideas político-religiosas ${ }^{34}$ le hicieron permanecer aislado ${ }^{35}$, sin discípulos directos que pudieran profundizar en su obra. Así, sus planteamientos tardaron más de dos décadas en ser aceptados de manera unánime. La obra de Cauchy, de gran complejidad, requería tiempo de reflexión y cierto sosiego para su asimilación, elementos de los que sin duda Vallejo nunca anduvo sobrado. La trascendencia de la obra del matemático francés, en la que se plantean nuevas vías de trabajo bajo nuevas perspectivas, necesitaba un análisis que, por su azarosa vida, Vallejo no pudo realizar.

Como se ha visto, los planteamientos que Vallejo usó para el desarrollo del cálculo diferencial en la segunda edición del cuarto volumen de su Tratado Elemental de Matemáticas (Vallejo, 1832) no permiten caracterizarla como obra explicada a partir de los planteamientos de Cauchy, pero esto no significa que no conociera su obra, pues las citas y referencias son abundantes, aunque selectivas. No obstante, la preponderancia que Vallejo otorga a los aspectos didácticos frente a los científicos posterga el desarrollo de Cauchy en favor de otros más fáciles de explicar y entender.

A este respecto, conviene recordar que la biografía de Vallejo muestra la vocación docente de su obra desde su acceso en 1802 a la cátedra del Seminario de Nobles de Madrid (Hernanz y Medrano, 1990, pp. 429-432). En este contexto debe entenderse el planteamiento de Vallejo ante las propuestas, no sólo de Cauchy, sino de cualquier avance en otra rama de las matemáticas.

Así, Vallejo, como Lacroix, evitó - por razones esencialmente didácticas - modificar sustancialmente los aspectos fundamentales de su cálculo infinitesimal, aunque sin renunciar a incorporar gran cantidad de notas muy actualizadas - que en ocasiones ocupan más espacio que el tema que está tratando. Probablemente este no fuera el mejor método para realizar esta segunda edición, que en ocasiones resulta difícil de seguir debido precisamente al gran número de notas a pie de página, pero dejó marcado el camino por el que el análisis matemático iba a discurrir.

Por otra parte, conviene destacar que Vallejo, a pesar de tener clara la finalidad docente de su texto, no renunció al rigor matemático y expositivo ni por motivos didácticos ni en aras de la apertura de nuevas vías de investigación, lo que le sitúa inequívocamente en la línea de desarrollo matemático del siglo XIX, durante el cual se produjo una vuelta generalizada al rigor.

A este respecto cabe recordar que el proceso de pérdida de interés por el rigor que se había producido en las matemáticas del siglo XVIII, justificado en aras de una mayor facilidad de divulgación o de apertura de nuevas vías de investigación, había comenzado ya en el siglo XVII: las ataduras a la búsqueda de nuevas soluciones que imponían los presupuestos griegos había provocado un paulatino abandono del rigor en virtud de las expectativas generadas por los nuevos métodos y resultados. Los métodos de la matemática griega, especialmente admirada por su alto grado de rigor, «no eran heurísticos; no se adaptaban bien a sugerir ideas sobre cómo atacar un problema nuevo» (Andersen, 1984, p. 24). Pero en el siglo XIX se produce una reacción, en sentido contrario, de revisión de conceptos e ideas anteriormente considerados evidentes: todo debe ser demostrado, nada debe quedar a expensas de la intuición; se demuestran los teoremas de valor medio - evidentes a la vista de los matemáticos del siglo XVIII-, se rechaza toda alusión a la velocidad o el movimiento -donde están implícitas ideas relacionadas con la continuidad sin ser explicitadas ni demostradas. En cuanto a la fundamentación del análisis matemático, esta tendencia ha sido definida como una retirada de la frontera de lo "obvio» (Grabiner, 1990, p. 173), en la que Cauchy ha sido tradicionalmente considerado más riguroso que sus inmediatos predecesores, aunque con matices (Cauchy, 1994, pp. 13-17) y discrepancias (Grattan-Guinness, 1990, vol. 2, pp. 694-695).

Vallejo, que practicó una presentación detallada de las deducciones que facilitara el paso de un eslabón a otro de la cadena de razonamiento y evitó plantear proposiciones como axiomas o dar por evidente lo que no lo era, manifestó repetidamente su total sintonía con esta tendencia, singularmente en una crítica a muchos libros franceses escritos durante el periodo revolucionario:

Donde había un barranco que saltar, un obstáculo que vencer, un defecto de la ciencia, porque no se hallase suficientemente adelantada, ó porque el autor no estuviese en disposición de explicarla como correspondía, echaba mano de un es claro, es evidente, se viene á los ojos, es fácil de conocer, ó de otras expresiones equivalentes, con las cuales deslumbraban al lector: y á veces les decían que eran claras y evidentes, no solo cosas que están muy lejos de serlo, sino también proposiciones dudosas y aun proposiciones falsas (Vallejo, 1821, pp. IV-V). 
En cualquier caso, las sensibilidades compartidas de Vallejo entre la didáctica y el rigor muestran su capacidad para asimilar las dos preocupaciones principales de las matemáticas del siglo XIX en cuanto a docencia e investigación, en un personalísimo proceso dinámico de actualización de conocimientos que, en

\section{NOTAS}

1 Para su biografía véase (Hernanz y Medrano, 1990) y (Gentil Baldrich, 1999).

2 Las interacciones entre matemáticas y política en el primer tercio del siglo XIX español son complejas, hasta el punto de que las situaciones de represión o exilio político de diferentes autores se traducen en una intensificación de su producción matemática. A este respecto véase (Hormigón, 1995).

3 Aunque ha sido abordada desde la perspectiva de la investigación histórica en educación matemática en (Maz, Torralbo y Rico, 2006) y (López Flores, 2011).

4 Un estudio en esta Memoria desde el punto de vista de la geometría diferencial puede verse en (Garma, 1994, pp. 230-235).

5 La figura de Lacroix, de gran influencia en España y otros países de su entorno europeo, es un ejemplo claro del perfil profesional que se empezó a gestar en esta época, a saber, el matemático profesor y autor de libros de texto de gran difusión e influencia (Ehrhardt, 2009). Lacroix se dedicó a la enseñanza de las matemáticas a lo largo de toda su vida en centros tan destacados como la École d'Artillerie, École Centrale des Quatre Nations, École Normale de I'an III, École Polytechnique, Lycée Bonaparte y Faculté des Sciences de París. Destacó en la elaboración de varios tratados sobre matemáticas, con los que consiguió fama. Sus libros de texto, dirigidos a un nivel medio-superior, abarcan toda la matemática en un esfuerzo por compendiar y resumir todos los puntos de vista más destacados, muy al estilo enciclopedista, tan en boga entonces. En un periodo en el que estaban en marcha las reformas educativas resultantes de la Revolución Francesa, Lacroix no sólo intentó componer manuales que recogieran los elementos más originales, sino también estructurarlos para facilitar en lo posible el acceso a un público amplio, mediante una presentación coherente y de fácil lectura acompañada de un análisis detallado sobre los fundamentos de cada rama y materia. El éxito de su obra fue tal que alcanzó varias ediciones y fue traducida a diferentes idiomas (alemán, español, inglés, italiano, portugués).

6 Este trabajo omite la consideración del cálculo infinitesimal en el Compendio de Vallejo (Vallejo, 1819, vol. 2, pp. 44-146) por tratarse de una versión abreviada de los contenidos de la primera edición de su Tratado.

7 Los 7 volúmenes de lo que a partir de 1805 se conocería como Cours élémentaire de mathématiques pures, à l'usage de l'École centrale des Quatre-Nations de Lacroix, recomendado en los liceos franceses, habían sido publicados en el de- cuanto al desarrollo del análisis matemático, no quedó significativamente arraigado en España, en buena parte debido a la falta de condiciones institucionales y profesionales que permitieran ejercer el oficio de matemático en el sentido moderno de la palabra (Ausejo, 1998, pp. 215-224).

cenio inmediatamente anterior (1795-1802). Sobre qué volúmenes eran considerados verdaderamente elementales por Lacroix véase (Caramalho Domingues, 2008, pp. 283-285).

8 Vallejo presenta exactamente el mismo enfoque -con las mismas expresiones- que Chaix, que afirma: "pero si el punto A mudase á cada instante la direccion de su movimiento (...) la línea ABCDE será curva" (Chaix, 1801, p. 98).

9 Esta forma de denotar las funciones, que convive en la Memoria con la notación actualmente usual cuando deriva -i.e. $z^{\prime}=f(x+k)$-, será sustituida en su Tratado por $z=f .(x) ; u=f .(t)$; en la segunda edición del Tratado el punto entre la $f$ y el paréntesis empieza a desaparecer (Vallejo, 1832, p. 154).

10 Ya que Chaix sostiene que $d y / d x$ debe considerarse como un símbolo único, no como cociente de diferenciales. Cuando usa el término diferenciar en sus Instituciones (Chaix, 1801), en realidad, deriva.

11 Derivadas en el caso de Lagrange.

12 Para entonces ya resuelta (Cauchy, 1821, pp. 45-69). Vallejo disculpó veladamente su error de juventud citando a (Jephson, 1826, p. 200), que obviamente sólo era responsable de sus propios errores (Vallejo, 1832, p. 325).

13 De su éxito da cuenta la fecha de publicación de la segunda edición revisada y aumentada (Lacroix 1806), que sólo presenta un par de cambios significativos respecto de la primera (Caramalho Domingues, 2008, p. 309).

14 Así se explica claramente en (Lacroix, 1810, p. 146).

15 Las críticas se refieren fundamentalmente a la dificultad de su uso en las diferenciales de funciones de varias variables. Posteriormente hará un estudio comparativo de las diferentes notaciones propuestas (Lacroix, 1810, pp. 242ss.).

16 La fórmula del binomio sólo se había demostrado para valores enteros y racionales. Considerar su validez para todo incremento de $x$ implica de una u otra forma el concepto de continuidad y una estructura de número real de la que se carecía.

17 El límite -en el sentido que se daba entonces a este paso al límite- del cociente incremental.

18 Cuestión no intrascendente cuya solución fue abordada por todos los autores importantes. 
19 Párrafo enfatizado en cursiva en el original.

20 "Le calcul différentiel fournit de méthodes très expéditives pour développer les fonctions en séries" (Cauchy, 1821, p. 164).

21 "Que les principes du calcul différentiel, et ses applications les plus importantes, peuvent être facilement exposés, sans l'intervention des séries" (Cauchy, 1823, p. vj).

22 (Vallejo, 1832, pp. xii, xiv, xv, 12, 38, 81, 87, 100, 107, 195, $338,368,457,467$ ss., 495). A estas referencias hay que añadir aquellas en las que toma algún concepto del autor francés sin citarlo, por ejemplo en la parte dedicada a continuidad, como se verá más adelante.

23 (Vallejo, 1832, pp. xv, 48, 83, 107, 194, 338, 457, 494, 495). No obstante, hay que tener en cuenta que el texto de la primera edición está fuertemente inspirado en Lacroix, por lo que la influencia no citada de este autor en esta segunda edición sigue siendo mucho mayor de lo que el número de citas explícitas indica.

24 (Vallejo, 1832, pp. xiv, xv, 38, 51, 457, 474, 477).

25 (Vallejo, 1832, pp. 49, 176, 299, 457, 495, 495).

26 (Vallejo, 1832, pp. 165, 176, 194, 202, 299, 495).

27 También son varias las veces que se refiere a Cauchy para ampliar los temas en el campo de los números complejos que, por razones de espacio, queda fuera de este estudio.

\section{BIBLIOGRAFÍA}

Andersen, Kirsti (1984), "Las técnicas del cálculo”. En: GrattanGuinness, Ivor (comp.), Del cálculo a la teoría de conjuntos, 1630-1910. Una introducción histórica, Madrid, Alianza Editorial, pp. 22-68.

Ausejo, Elena (1998), “El oficio de matemático en la Edad Contemporánea (1808-1936)”. En: Español, Luis (ed.), Matemática y región, Logroño, Instituto de Estudios Riojanos, 211226.

Ausejo, Elena y Francisco Javier Medrano Sánchez (2012), “La fundamentación del Calculus en España: el Cálculo Infinitesimal en Gabriel Ciscar (1760-1829)", Llull, Revista de la SEHCYT, 35 (76), pp. 305-316.

Ausejo, Elena y Francisco Javier Medrano Sánchez (2015), “Jorge Juan y la consolidación del cálculo infinitesimal en España (1750-1814)", En: Alberola, Armando; Die, Rosario; Mas, Cayetano (eds.), Jorge Juan Santacilia en la España de la Ilustración, Alicante, Publicaciones Universidad de Alicante / Casa de Velázquez.

Caramalho Domingues, João (2008), Lacroix and the Calculus. Basel, Birkhäuser.
28 Aunque la idea aparece ya, por ejemplo, en la Mémoire sur les intégrales définies prises entre des limites imaginaires de 1825.

29 Obra en cuatro volúmenes. La primera memoria sobre el cálculo de residuos se titula Sur un nouveau genre de calcul analogue au Calcul infinitésimal (Cauchy, 1882-1974, 2a ser., tomo VI, pp. 23-37)

30 Nótese que define una propiedad local, no puntual, puesto que se trata de continuidad entre unos límites, no en un punto (Cauchy, 1994, p. 90n).

31 Entresacado de (Cauchy, 1821, pp. 34ss.)

32 "voisinage" es la palabra usada en (Cauchy, 1821, p. 35), que es traducida por "en la inmediación" en (Vallejo, 1832, p. 154) y por "vecindad" en (Cauchy, 1994, p. 91).

33 Expresión usada en (Cauchy, 1821, p. 35).

34 Que quedan reflejadas en esta frase a propósito de su estado de ánimo durante la restauración borbónica: "all was in order in the world, with God on his catholic throne and the Bourbons at Versailles" (Grattan-Guinness, 1990, vol. 2, p. 641).

35 "The learned and industrious author of these writings [se refiere a Cauchy] does not count on numerous readers, no doubt; a general desertion seems to condemn his work to uselessness". Citado en (Grattan-Guinness, 1990, vol. 2, p. 641).
Cauchy, Augustin-Louis (1821), Cours d'Analyse, première partie, L'Analyse Algébrique. Paris, Imprimerie Royale.

Cauchy, Augustin-Louis (1823), Résumé des leçons données à l'École Royale Polytechnique sur le calcul infinitésimal. Paris, Imprimerie royale.

Cauchy, Augustin-Louis (1882-1974), CEuvres Complètes d'Augustin Cauchy. Paris, Gautier-Villars.

Cauchy, Augustin-Louis (1994), Curso de Análisis, Traducción y notas de Carlos Álvarez, introducción de Jean Dhombres. México, UNAM.

Chaix, José (1801), Instituciones de cálculo diferencial é integral con sus aplicaciones principales á las matemáticas puras y mixtas. Madrid, Imprenta Real.

Ehrhardt, Caroline (2009), “L'identité sociale d’un mathématicien et enseignant: Sylvestre-François Lacroix (17651843)", Histoire de l'éducation, 123, pp. 5-43, [en línea], doi: 10.4000/histoire-education.2024, disponible en: http://histoire-education.revues.org/2024 [consultado el 6/8/2014]. 
Garma, Santiago (1994), "Influencia de los matemáticos franceses en los matemáticos españoles a finales del siglo XVIII". En: Garma, Santiago; Flament, Dominique; Navarro, Víctor (eds.), Contra los titanes de la rutina, Madrid, Comunidad de Madrid/CSIC, pp. 209-235.

Gentil Baldrich, José María (1999), “Nuevos datos sobre la vida y la obra de José Mariano Vallejo y Ortega (1779-1846)", LluII, Revista de la SEHCYT, 22 (44), pp. 381-404.

Grabiner, Judith V. (1990), The Calculus as Algebra. J.-L. Lagrange 1736-1813. New York \& London, Garland Publishing.

Grattan-Guinness, Ivor (1990), Convolutions in French Mathematics, 1800-1840. Basel-Boston-Berlin, Birkhäuser Verlag.

Hernanz Pérez, Carlos y Francisco Javier Medrano Sánchez (1990), "José Mariano Vallejo: notas para una biografía científica", Llull, Revista de la SEHCYT, 13 (25), pp. 427-446.

Hormigón, Mariano (1995), “Les mathématiciens dans la vie politique espagnole pendant la première moitié du XIX siècle", Bolletino di Storia Scienze Matematiche, 15 (1), pp. 27-47.

Jephson, Thomas (1826), The Fluxional Calculus. An Elementary Treatise, designed for the Students of the Universities, and for those who desire to be acquainted with the principles of Analysis. London, Baldwin, Cradock and Joy.

Lacroix, Sylvestre-François (1797-1800), Traite du calcul différentiel et du calcul intégral, 3 vols. Paris, Chez Duprat.

Lacroix, Sylvestre-François (1802), Traité élémentaire de calcul différentiel et de calcul intégral. Précédé de réflexions sur la manière d'enseigner les Mathématiques, et d'apprécier dans les examens le savoir de ceux qui les ont étudiées. Paris, Chez Duprat.

Lacroix, Sylvestre-François (1806), Traité élémentaire de calcul différentiel et de calcul intégral, $2^{\text {ème }}$ édition, revue et corrigée. Paris, Chez Courcier.

Lacroix, Sylvestre-François (1810), Traite du calcul différentiel et du calcul intégral, vol. I. Paris, Chez Courcier.
López Flores, José Iván (2011), Un análisis sistémico de la obra de José Mariano Vallejo desde la perspectiva de la investigación histórica en educación matemática. Universidad de Salamanca, Tesis Doctoral.

Maz, A.; Torralbo, M.; Rico, L. (eds.) (2006), José Mariano Vallejo, el matemático ilustrado. Una mirada desde la Educación Matemática. Córdoba, Servicio de Publicaciones de la Universidad de Córdoba.

Medrano, Francisco Javier (1998), "El cálculo diferencial en el Tratado Elemental de Vallejo". En: García Hourcade, Juan Luis; Moreno Yuste, Juan M.; Ruíz Hernández, Gloria (coords.), Estudios de Historia de las Técnicas, la Arqueología industrial y las ciencias. Actas del VI Congreso de la Sociedad Española de Historia de las Ciencias y de las Técnicas, Salamanca, Junta de Castilla y León, pp. 953-964.

Vallejo, José Mariano (1807), Memoria sobe la curvatura de las líneas en sus diferentes puntos, sobre el radio de curvatura y sobre las evolutas. Madrid, Imprenta de Tomás Albán.

Vallejo, José Mariano (1812-1817), Tratado Elemental de Matemáticas, escrito de órden de S. M. para uso de los caballeros seminaristas del Real seminario de nobles y demás casas de educación del reyno. Mallorca, en la Imprenta de Melchor Guasp, 3 tomos en 5 vols. (tomo II, 1a parte impreso en 1813; tomo II, 2a parte impreso por Felipe Guasp en 1813; tomo III impreso en Valencia por Estévan en 1817).

Vallejo, José Mariano (1819), Compendio de Matemáticas Puras y Mistas. Valencia, En la Imprenta de Estévan.

Vallejo, José Mariano (1821), Tratado Elemental de Matemáticas. Tomo I, parte I, 3a edición. Barcelona, Imprenta del Gobierno Político Superior.

Vallejo, José Mariano (1825), Tratado Elemental de Matemáticas, Tomo I, parte II, 3ạ edición. Madrid, Imprenta que fué de García.

Vallejo, José Mariano (1832), Tratado Elemental de Matemáticas, Tomo II, parte II, 2a edición. Madrid, Imprenta de D. Miguel Burgos. 